\title{
Neuroprotective effect of tanshinone IIA weakens spastic cerebral palsy through inflammation, p38MAPK and VEGF in neonatal rats
}

\author{
WEN-LUO ZHANG, YUE-AN CAO, JING XIA, LI TIAN, LU YANG and CHAO-SHENG PENG \\ Department of Special Medical Division, Navy General Hospital, Beijing 100048, P.R. China
}

Received August 3, 2016; Accepted August 14, 2017

DOI: $10.3892 / \mathrm{mmr} .2017 .8069$

\begin{abstract}
As one of main active ingredients of salvia miltiorrhizae, which is a traditional Chinese medicine, tanshinone IIA is the basis of its pharmacological activities. In the present study, the effect of tanshinone IIA on weakening spastic cerebral palsy (SCP) in neonatal rats was investigated. Radial arm water maze and holding tests were used to measure the alterations of spastic cerebral palsy, inflammation was measured using an ELISA kit, and western blot analysis was used to analyze the protein expression of p-p38 mitogen-activated protein kinase (MAPK) and vascular endothelial growth factor (VEGF). The central mechanisms involved in the mediation or modulation of inflammation, p-p38 MAPK and VEGF were also investigated. Treatment with tanshinone IIA effectively inhibited spastic cerebral palsy, and the activities of interleukin (IL)-1 $\beta$, IL-6, tumor necrosis factor- $\alpha$, monocyte chemoattractant protein 1, cyclooxygenase-2 and prostaglandin E2 in a neonatal rat model of SCP. Tanshinone IIA effectively suppressed the protein expression of inducible nitric oxide synthase (NOS), phosphorylated (p-) nuclear factor (NF)- $\kappa \mathrm{B}$, p-p38MAPK and VEGF, and activated the phosphorylation of inhibitor of $\mathrm{NF}-\kappa \mathrm{B}$ and the protein expression of neuronal NOS in the SCP rat model. These results suggested that the neuroprotective effect of tanshinone IIA weakened SCP through inflammation, p38MAPK and VEGF in the neonatal rats.
\end{abstract}

\section{Introduction}

Spastic cerebral palsy (SCP) is a common complication in premature infants. With the rapid progression of perinatology and technology in neonatal intensive care units, the survival

Correspondence to: Dr Chao-Sheng Peng, Department of Special Medical Division, Navy General Hospital, 6 Fucheng Road, Beijing 100048, P.R. China

E-mail: pengcscsbj@163.com

Key words: tanshinone IIA, spastic cerebral palsy, inflammation, p38 mitogen-activated protein kinase, vascular endothelial growth factor rates of premature infants have increased (1). However, $\sim 10-20 \%$ of premature infants are affected by sequelae in the nervous system at differing levels of severity, placing a substantial burden on families and society (2). Therefore, early diagnosis and prevention is urgently required (3). In addition to premature delivery, the occurrence of cerebral injury in premature infants is also correlated with hypoxia-ischemia, infection, oxidative stress and inflammatory response. Previous studies have demonstrated that inflammatory mediators and cytokines are involved in the pathophysiological process of SCP $(2,3)$. There has been an increasing focus on the changes of inflammatory factors when SCP is present (4).

As an important component of immune responses, the inflammatory response is closely associated with SCP (5). The inflammatory response includes the adhesion, metastasis, infiltration and activity of inflammatory cells, and the release of inflammatory cytokines (6). Inflammatory cytokines, including interleukin (IL)-6 and tumor necrosis factor (TNF)- $\alpha$, are involved in the entire process of the inflammatory response. The levels of inflammatory cytokines in children with hypoxic-ischemic brain damage are high, indicating that infection and hypoxic-ischemic brain damage may result in brain tissue damage through inflammatory factors (7). Increasing studies have demonstrated that high levels of proinflammatory cytokines in amniotic fluid, plasma or the umbilical cord blood may be associated with periventricular leukomalacia and subsequently lead to the development of SCP (6).

The expression of vascular endothelial growth factor (VEGF) is involved in the entire process of nervous system development (8). VEGF is expressed predominantly in surrounding regions of the neuroderm ventricle, which decrease from the inside to the outside. With the maturity of the central nervous system, the expression of VEGF decreases gradually. VEGF can reduce the apoptosis of endothelial cells and prevent the disappearance of blood capillaries. It can also induce the relaxation of angio-smooth muscle through the release of nitric oxide (NO) to protect ischemic brain tissues. Animal experiments have shown that, following cerebral damage the expression of VEGF can promote angiogenesis, resist apoptosis and protect neurocytes. However, the animals involved in this study were adults (9).

With the continuous progression of modern pharmacology, monomer compositions of various types of traditional 
Chinese medicine have been shown to have inhibitory effects on pulmonary interstitial fibrosis (10). Extracted from the classic and traditional Chinese medicine salvia miltiorrhizae, tanshinone IIA is a fat-soluble active constituent, which is used for treating cardiovascular disease (11). With a definite molecular structure, it is an active ingredient with the highest stability at a higher content (11). Experiments have shown that tanshinone IIA possesses several pharmacological activities, including oxygen radical scavenging, and anti-inflammatory and antitumor effects $(12,13)$. Therefore, in the present study, the neuroprotective effects of tanshinone IIA on the weakening of SCP were investigated in neonatal rats. The central mechanisms involved in the mediation or modulation of inflammation, p38 mitogen-activated protein kinase (MAPK) and VEGF were also investigated.

\section{Materials and methods}

Animals and SCP model. A total of 26 male Sprague-Dawley specific-pathogen-free rats (7-day-old, 15-20 g) were provided by the Navy General Hospital Experimental Animal Center (Beijing, China). The rats were housed at $22-23^{\circ} \mathrm{C}$ and $50-55 \%$ humidity with a 12-h light/dark cycle, and ad libitum access to pellet chow and water. The animals were randomly assigned into three groups $(n=26)$ : Sham injury (skin incision of the skull only; $n=6$ ), SCP model (SCP rat treated with normal saline; $n=10$ ) and tanshinone IIA group (SCP rat treated with $20 \mathrm{mg} / \mathrm{kg}$ of tanshinone IIA for 5 weeks; $\mathrm{n}=10$ ).

Unilateral occlusion of the carotid artery was performed to establish the SCP animal model. The 7-day-old rats were used as the SCP animal model to represent what occurs in newborn humans. The rats were anesthetized via an intraperitoneal injection of $10 \%$ chloral hydrate and placed in a supine position. A median neck incision was performed and the left common carotid artery was ligated. The wound was then sterilized and sutured, and the animals were placed in cages under routine conditions. The animal experiments were approved by the Ethics Committee of the Naval General Hospital (Beijing, China).

Radial arm water maze test and holding test. The eight-arm radial maze consisted of a central platform symmetrically extended. The rats were deprived of water for $48 \mathrm{~h}$ prior to assessment. A single well was placed at the outer end of each arm and the rat was allowed to drink for $30 \mathrm{~min}$. The rat was allowed to freely explore the maze containing the water-filled wells for 2 days prior to the spatial discrimination test days. In the spatial discrimination test, the wells were baited in only three arms and the sequence of angles was $135^{\circ}, 90^{\circ}$ and $135^{\circ}$.

Each rat was measured over three daily sessions consisting of five trials separated at 1-min intervals. The rat was placed on the central platform in each trial, facing arm three, and the experiment ended when the rat visited the three baited arms. Three measurements were recorded: i) time taken to visit the three baited arms; ii) number of re-entries into previously visited baited arms; iii) number of entries into a non-baited arm. In the holding test, the forepaws of the rat were grasped in a hollow plastic tube, which was placed horizontally from a desk to allow them to hold freely. The time spent suspended during $1 \mathrm{~min}$ was recorded.

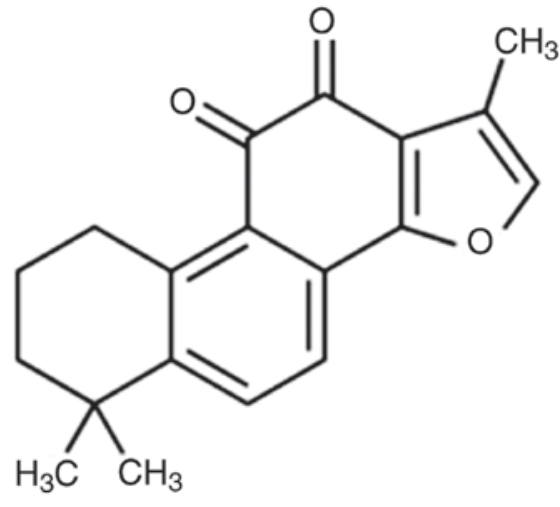

Figure 1. Chemical structure of tanshinone IIA.

ELISA analysis. The hippocampus from every rat was dissected and homogenized with ice-cold radioimmunoprecipitation (RIPA) and $0.1 \mathrm{mmol} / 1$ of PMSF for $30 \mathrm{~min}$. The concentration of total protein was determined using Bio-Rad protein assay reagent (Bio-Rad Laboratories, Inc., Hercules, CA, USA). Following determination, $110 \mu \mathrm{g}$ total protein was incubated with TNF- $\alpha$ (cat. no. E-EL-R0019c), interleukin (IL)-1 $\beta$ (cat. no. E-EL-R0012c), IL-6 (cat. no. E-EL-R0015c) and monocyte chemoattractant protein 1 (MCP-1; cat. no. E-EL-R0633c) ELISA kits (Elabscience Biotechnology Co., Ltd., Bethesda, MD, USA) at $37^{\circ} \mathrm{C}$ for $1 \mathrm{~h}$. The absorbance was measured using an ELISA analyzer (DNM-9606; Perlong Medical, Jiangsu, China) at $450 \mathrm{~nm}$.

Western blot analysis. The hippocampus from every rat was dissected and homogenized with ice-cold RIPA and $0.1 \mathrm{mmol} / 1$ of PMSF for $30 \mathrm{~min}$. The concentration of total protein was determined using Bio-Rad protein assay reagent, and $50 \mu \mathrm{g} /$ lane total protein was subjected to $8-10 \%$ SDS-polyacrylamide gel electrophoresis, following which it was electrotransferred onto a nitrocellulose membrane. The nitrocellulose membrane was blocked with $5 \%$ skim milk powder in TBST and probed consecutively with the following primary polyclonal antibodies: Nuclear factor (NF)-кB (cat. no. sc-109; 1:500; Santa Cruz Biotechnology, Inc., Dallas, TX, USA), phosphorylated (p)-p38MAPK (cat. no. sc-17852-R; 1:500; Santa Cruz Biotechnology, Inc.), VEGF (cat. no. sc-13083; 1:500; Santa Cruz Biotechnology, Inc.), p-inhibitor of NF- $\kappa$ B (p-IкB; cat. no. sc-101713; 1:500; Santa Cruz Biotechnology, Inc.), inducible nitric oxide synthase (iNOS; cat. no. sc-649; 1:500; Santa Cruz Biotechnology, Inc.), neruronal NOS (nNOS; cat. no. sc-8309; 1:500; Santa Cruz Biotechnology, Inc.) and $\beta$-actin (cat. no. sc-7210; 1:500; Santa Cruz Biotechnology, Inc.) at $4^{\circ} \mathrm{C}$ overnight. The membrane was then washed with TBST and incubated with the anti-rabbit immunoglobulin $\mathrm{G}$ secondary antibody (1:5,000; cat. no. sc-2004; Santa Cruz Biotechnology Inc.) at $37^{\circ} \mathrm{C}$ for $1 \mathrm{~h}$. Protein expression was visualized using BeyoECL Plus (Beyotime Institute of Biotechnology, Haimen, China) and analyzed using Image_Lab_3.0 software (Bio-Rad Laboratories, Inc.).

Statistical analysis. Data are expressed as the mean \pm standard deviation using SPSS 17.0 software (SPSS, Inc., 

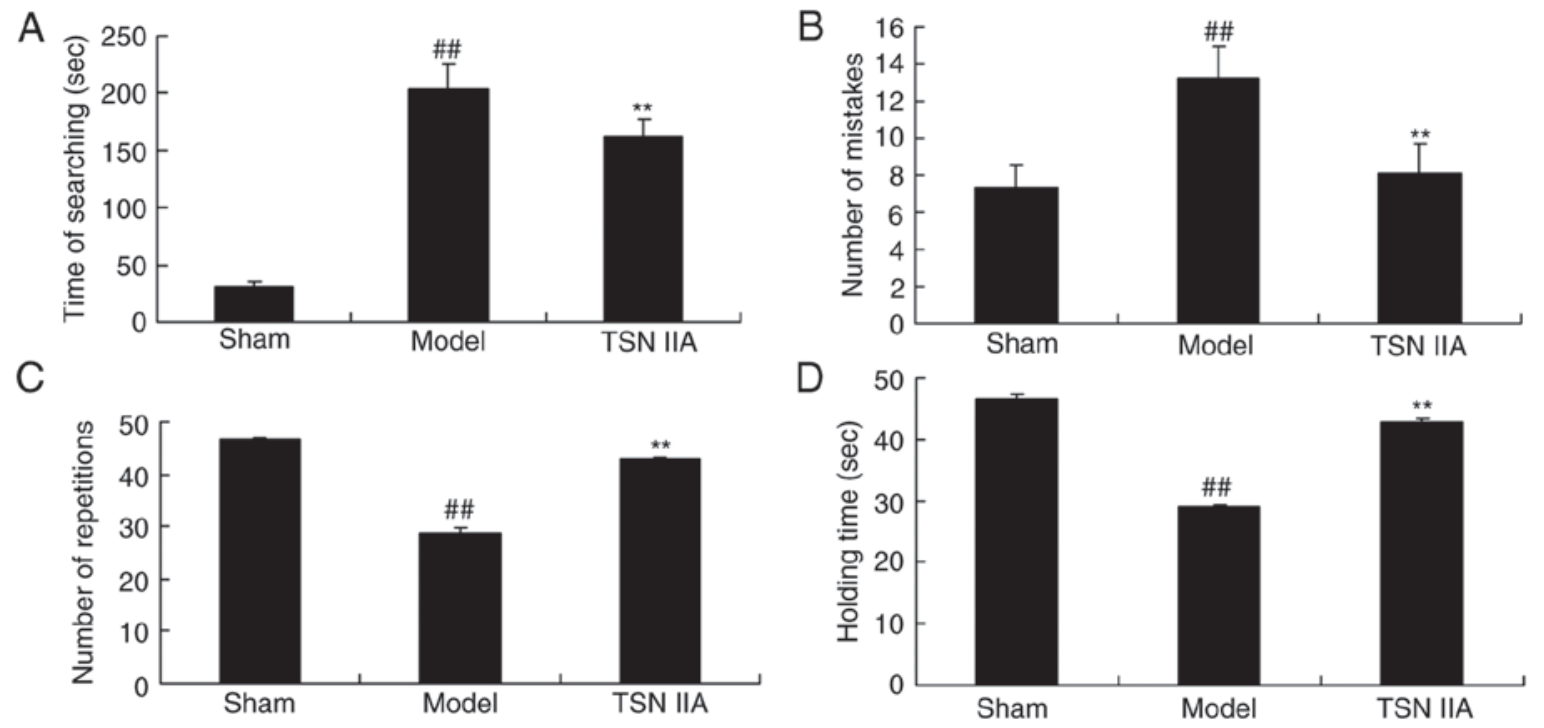

Figure 2. Neuroprotective effect of tanshinone IIA weakens SCP. The neuroprotective effect of tanshinone IIA reduced the (A) time spent searching and the (B) number of mistakes, and increased the (C) number of repetitions and (D) holding time of the SPC rats. ${ }^{\# *} \mathrm{P}<0.01$ vs. the sham group; ${ }^{* *} \mathrm{P}<0.01 \mathrm{vs}$. the model group. Sham, sham control; Model, SPC model; TSN IIA, tanshinone IIA; SPC, spastic cerebral palsy.
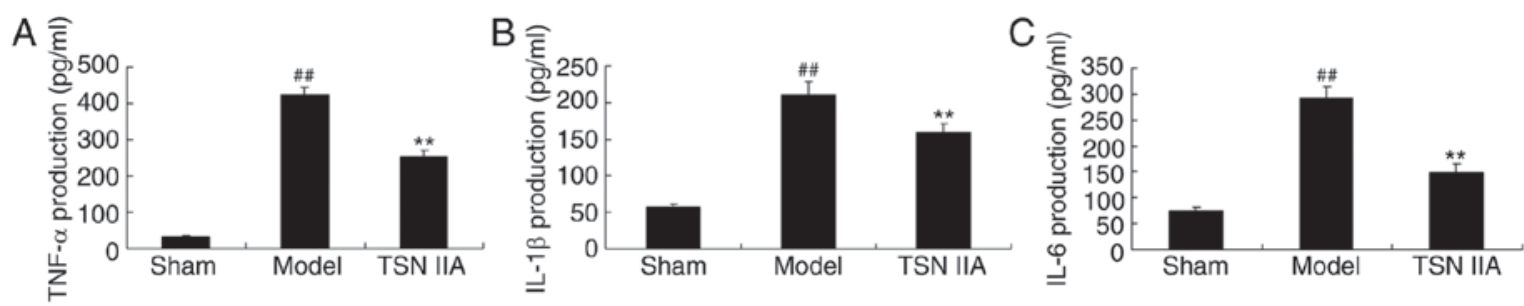

Figure 3. Neuroprotective effect of tanshinone IIA reduces the expression of inflammatory factors. The neuroprotective effect of tanshinone IIA reduced the expression of (A) TNF- $\alpha$, (B) IL-1 $\beta$ and (C) IL- $6 .{ }^{\# \#} \mathrm{P}<0.01$ vs. the sham group; ${ }^{* *} \mathrm{P}<0.01$ vs. the model group. SPC, spastic cerebral palsy; sham, sham control; Model, SPC model; TSN IIA, tanshinone IIA; TNF- $\alpha$, tumor necrosis factor- $\alpha$; IL, interleukin.

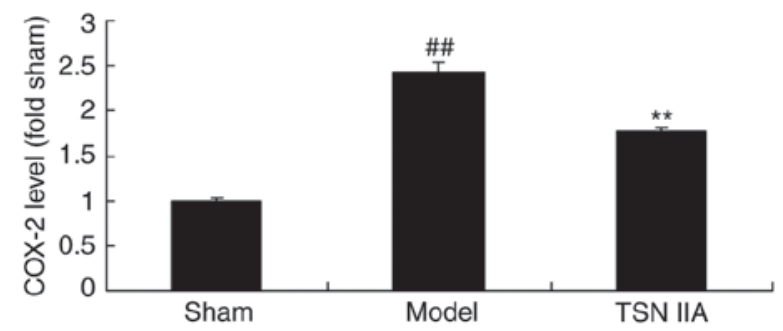

Figure 4. Neuroprotective effect of tanshinone IIA reduces the expression of COX-2. The expression of COX-2 was reduced by tanshinone IIA. ${ }^{\# \#} \mathrm{P}<0.01$, compared with the sham group; ${ }^{* *} \mathrm{P}<0.01$ vs. the model group. SPC, spastic cerebral palsy; sham, sham control; Model, SPC model; TSN IIA, tanshinone IIA; COX-2, cyclooxygenase 2.

Chicago, IL, USA). The experimental results were evaluated using one-way analysis of variance by a Tukey post-hoc test. $\mathrm{P}<0.05$ was considered to indicate a statistically significant difference.

\section{Results}

Neuroprotective effect of tanshinone IIA reduces SCP. The structural formula of tanshinone IIA is shown in Fig. 1. As exhibited in Fig. 2A and B, there was a significant increase

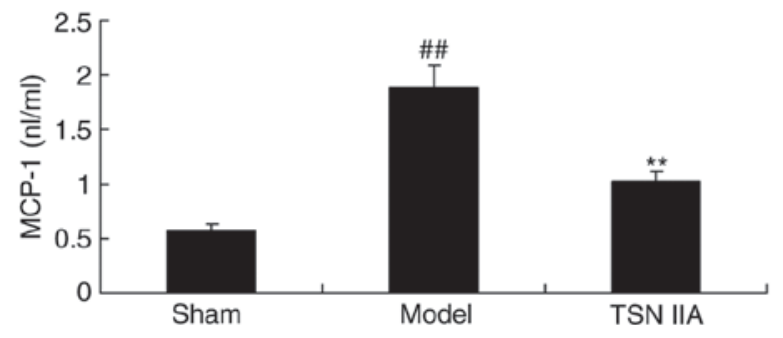

Figure 5. Neuroprotective effect of tanshinone IIA reduces the expression of MCP-1. The expression of MCP-1 was reduced by tanshinone IIA. ${ }^{\# \#} \mathrm{P}<0.01$, compared with the sham group; ${ }^{* *} \mathrm{P}<0.01$ vs. the model group. SPC, spastic cerebral palsy; sham, sham control; Model, SPC model; TSN IIA, tanshinone IIA; MCP-1, monocyte chemoattractant protein 1.

in the time spent searching and number of mistakes in the SCP model group rats, compared with the control group rats. Significant decreases in the number of repetitions and holding times were observed in rats of the SCP model group, compared with those of the control group (Fig. 2C and D). Treatment with tanshinone IIA significantly inhibited the increased search duration and number of mistakes, and increased the inhibited number of repetitions and holding times in the SCP rats, compared with the rats in the SCP model group (Fig. 2A and D). 
A

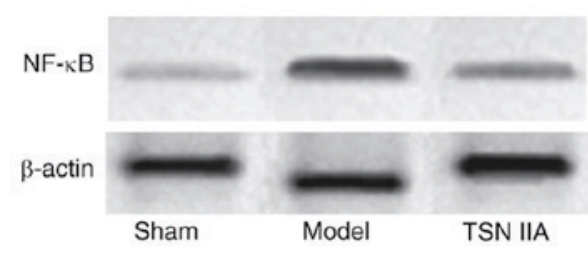

C

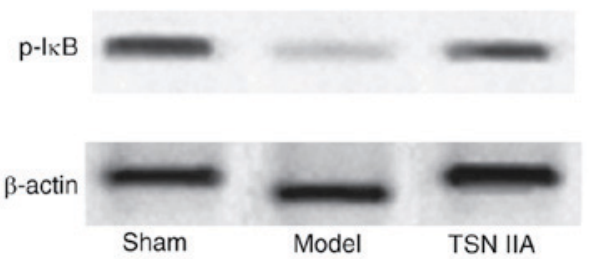

B

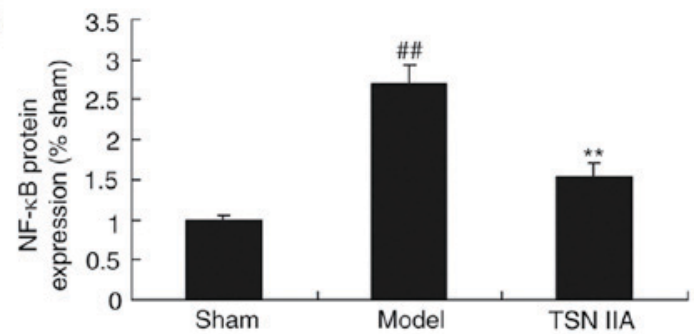

D

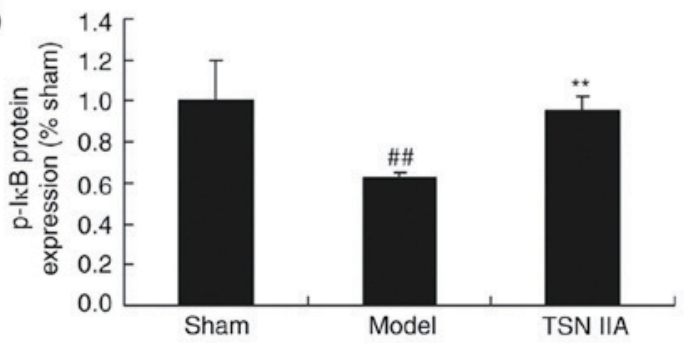

Figure 6. Neuroprotective effect of tanshinone IIA weakens the NF- $\mathrm{B}$ signaling pathway. Western blot analysis was used to determine protein expression

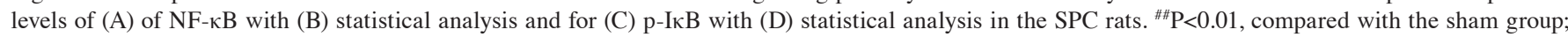
${ }^{* *} \mathrm{P}<0.01$ vs. the model group. SPC, spastic cerebral palsy; sham, sham control; Model, SPC model; TSN IIA, tanshinone IIA; NF- $\mathrm{B}$, NF-nuclear factor- $\kappa \mathrm{B}$; $\mathrm{p}-\mathrm{I} \kappa \mathrm{B}$, phosphorylated inhibitor of NF- $\mathrm{NB}$.

Neuroprotective effect of tanshinone IIA reduces the expression of inflammatory factors. To examine the anti-inflammatory effect of tanshinone IIA in the SCP rats, the levels of TNF- $\alpha$, IL-1 $\beta$ and IL- 6 in the hippocampal tissue samples were analyzed. The levels of TNF- $\alpha$, IL- $1 \beta$ and IL- 6 in the SCP rats were significantly increased, compared with those in the control group rats (Fig. 3A-C). Pre-treatment with tanshinone IIA significantly inhibited the increased levels of TNF- $\alpha$, IL-1 $\beta$ and IL-6 in the SCP rats, compared with the rats in the sham control group (Fig. 3A-C).

Neuroprotective effect of tanshinone IIA reduces the $m R N A$ expression of $C O X-2$. To further examine the anti-inflammatory effect of tanshinone IIA in the SCP rats, the expression levels of COX-2 were analyzed. The level of COX-2 was significantly increased in the SCP model, compared with that in the sham group. The administration of tanshinone IIA significantly inhibited the promoted expression level of COX-2 in the SCP rats, compared with the rats in the sham control group (Fig. 4).

Neuroprotective effect of tanshinone IIA reduces the expression of $M C P-1$. To examine the anti-inflammatory effect of tanshinone IIA in the SCP rat, the expression of MCP-1 was also measured. As demonstrated in Fig. 5, the expression if MCP-1 in the SCP model was significantly increased, compared with that in the sham group. Treatment with tanshinone IIA significantly reduced the expression of MCP-1 in the SCP rats, compared with the rats in the SCP model group (Fig. 5).

Neuroprotective effect of tanshinone IIA inhibits the $N F-\kappa B$ signaling pathway. To investigate the anti-inflammatory mechanism of tanshinone IIA in the SCP rats, the protein expression levels of $\mathrm{NF}-\kappa \mathrm{B}$ and $\mathrm{p}-\mathrm{I} \kappa \mathrm{B}$ were detected using western blot analysis. The results of the western blot analysis demonstrated that the protein expression levels of $N F-\kappa B$ and $\mathrm{p}-\mathrm{I} \kappa \mathrm{B}$ were significantly increased and decreased in the SCP model, respectively (Fig. 6A-D). Tanshinone IIA significantly suppressed the protein expression of $N F-\kappa B$ and increased the protein expression of $\mathrm{p}-\mathrm{I} \kappa \mathrm{B}$ in the SCP rats, compared with the rats in the SCP model group (Fig. 6A-D).

Neuroprotective effect of tanshinone IIA reduces the expression of $N O$. In order to examine the effect of tanshinone IIA on changes in NO levels, the protein expression levels of iNOS and nNOS were analyzed using western blot analysis. As exhibited in Fig. 7A-C, the protein expression of iNOS was increased and the protein expression of nNOS was reduced in the SCP model group, compared with the levels in the sham control group. Treatment with tanshinone IIA significantly inhibited the induced protein expression of iNOS and increased the suppressed protein expression of nNOS in the SCP rats (Fig. 7A-C).

Neuroprotective effect of tanshinone IIA reduces the protein expression of $p 38 M A P K$. The present study further examined the mechanism underlying the effects of tanshinone IIA in the SCP rats. p38MAPK may be involved in the effect of tanshinone IIA on SCP. As exhibited in Fig. 8A and B, the expression of p-p38 of the SCP rat group was significantly increased, compared with that in the sham group. This increased protein expression of p-p38 in the SCP rats was significantly suppressed by tanshinone IIA (Fig. 8A and B).

Neuroprotective effect of tanshinone IIA reduces the protein expression of VEGF. The present study investigated whether tanshinone IIA had an effect on blood vessels in the brain by detecting the protein expression of VEGF. The results indicated that the protein expression of VEGF was significantly suppressed in the SCP model group, compared with that in the sham control group. Treatment with tanshinone IIA 
A

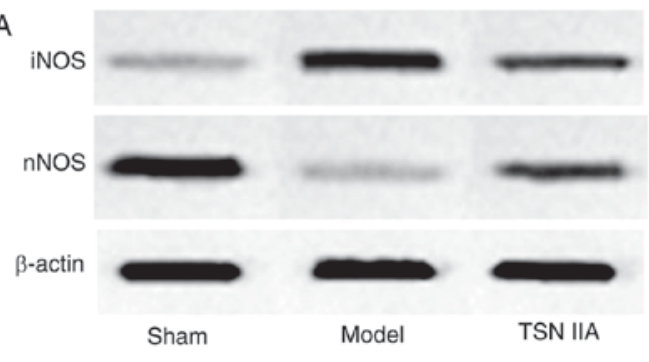

B

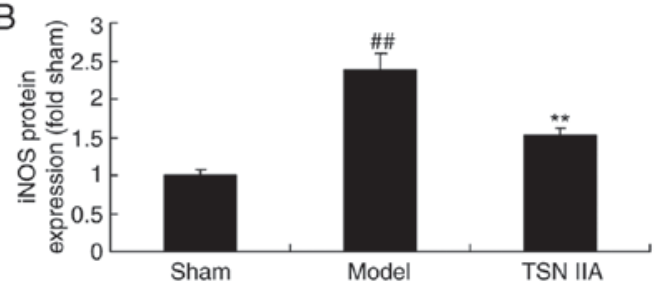

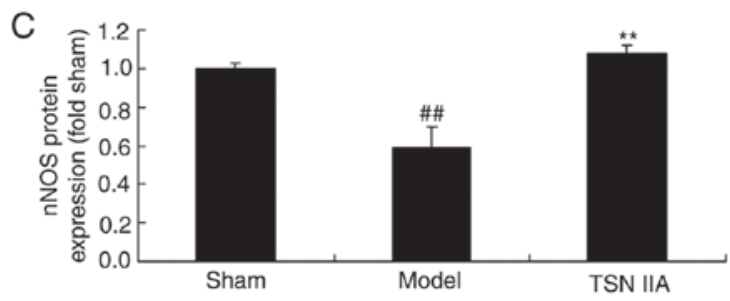

Figure 7. Neuroprotective effect of tanshinone IIA reduces the expression of NO. (A) Protein expression of iNOS and nNOS were examined using western blot analysis. Statistical analysis of the protein expression levels of (B) iNOS and (C) nNOS were performed in the SPC rats. ${ }^{\# /} \mathrm{P}<0.01$ vs. the sham group; ${ }^{* *} \mathrm{P}<0.01$ vs. the model group. SPC, spastic cerebral palsy; sham, sham control; Model, SPC model; TSN IIA, tanshinone IIA.
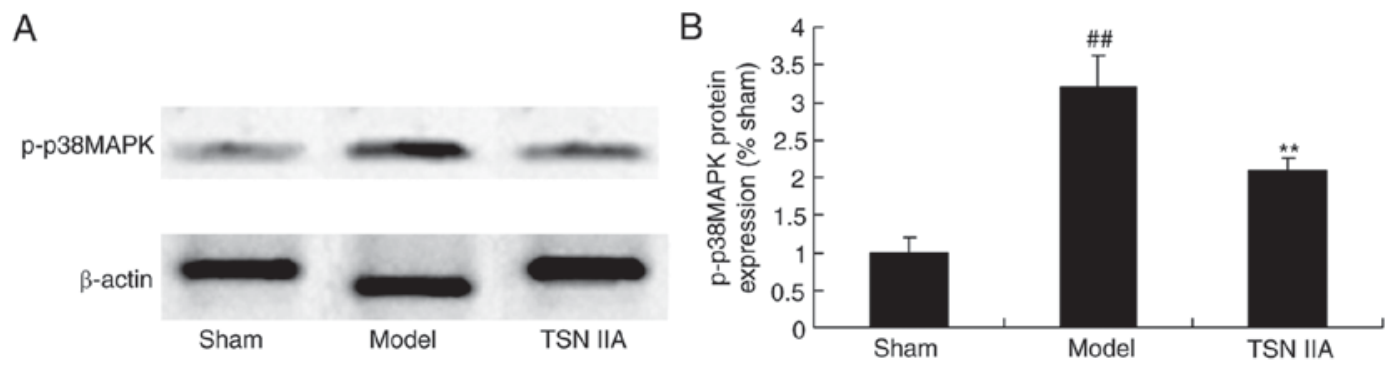

Figure 8. Neuroprotective effect of tanshinone IIA reduces the protein expression of p38MAPK. (A) Protein expression of p-p38MAPK was determined using western blot analysis, followed by (B) statistical analysis in the SPC rats. ${ }^{\# \#} \mathrm{P}<0.01$ vs. the sham group; ${ }^{* *} \mathrm{P}<0.01$ vs. the model group. SPC, spastic cerebral palsy; sham, sham control; Model, SPC model; TSN IIA, tanshinone IIA.
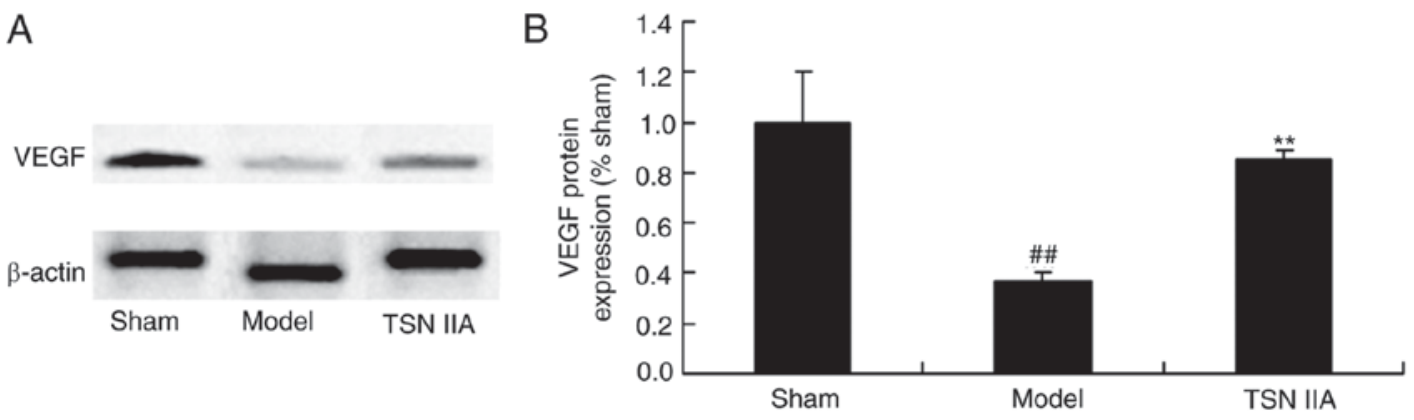

Figure 9. Neuroprotective effect of tanshinone IIA weakens the protein expression of VEGF. (A) Protein expression of VEGF was determined using western blot analysis, followed by (B) statistical analysis in the SPC rats. ${ }^{\# /} \mathrm{P}<0.01$ vs. the sham group; ${ }^{* *} \mathrm{P}<0.01$ vs. the model group. SPC, spastic cerebral palsy; sham, sham control; Model, SPC model; TSN IIA, tanshinone IIA.

significantly increased the protein expression of VEGF in the SCP group (Fig. 9A and B).

\section{Discussion}

SCP refers to the syndrome caused by non-progressive cerebral damage and developmental defects occurring between fertilization and infancy, which features dyskinesia and postural dysfunction (14). Brain-derived SCP, non-progressive cerebral damage and symptoms occur at infancy caused by complications, whereas central coordination disturbance caused by progressive disease and temporary motor retardation in normal children are excluded (15). In the present study, the tests performed showed that tanshinone IIA significantly inhibited the time spent searching and number of mistakes, and increased the number of repetitions and holding times in the SCP rats. 
Similar results have been demonstrated in previous animal experiments. When lipopolysaccharide was injected into the uterus of pregnant rats, the expression levels of TNF- $\alpha$ and IL-1 $\beta$ in the brains of newborn rats were dose-dependently increased (15). Fibrillary acidic protein was positive in the hippocampus and cortex, astrocytes were increased, myelin basic protein was deceased, and the activities of oligodendroglial cells showed abnormal changes (16). The results of the present study revealed that tanshinone IIA significantly inhibited the increased levels of TNF- $\alpha$, IL-1 $\beta$ and IL-6 in the SCP rats. Jiang et al also suggested that tanshinone IIA protects against folic acid-induced acute kidney injury through inhibition of the inflammatory response (17).

Monocytes, macrophages, endothelial cells and contractile fiber cells can express MCP-1 (18). Major biological effects of MCP-1 include the chemotaxis of monocytes, and it can act on lymphocytes and basophilic granulocytes, although it has no biological effects on neutrophile granulocytes (18). MCP-1 receptor is a member of the g-protein coupled receptor super-family (19). Following the combination of MCP-1 with its targeted specific receptor, it activates the specific receptor of MCP-1 through g-protein coupling on the cytomembrane (19). It also triggers the release of calcium ions in the cytoplasm and induces the activation of protein kinase C. In SCP, the injured cerebral tissues produce various inflammatory chemokine factors (20). MCP-1 is a major inflammatory chemokine factor, which exhibits potent chemotaxis of monocytes/macrophages (20). The monocytes/macrophages gather to regions of inflammation, and are involved in the occurrence and progression of the inflammatory response (21), which causes damage to brain tissues (21). The data obtained in the present study demonstrated that tanshinone IIA significantly weakened the expression levels of MCP-1 and COX-2, inhibited the induced protein expression of iNOS and increased the protein expression of nNOS in the SCP rats. Tang et al suggested that tanshinone IIA in neuropathic pain is mediated predominantly by the downregulation of the c-Jun N-terminal kinase/MCP-1 pathway (22).

$\mathrm{NF}-\kappa \mathrm{B}$ comprises a group of transcription factors in eukaryocytes, which is distributed widely in the nervous system (23). Under physiological conditions, it does not have transcriptional activities. When the cells are stimulated, its phosphorylation and ubiquitylation are initiated through the secondary messenger system (24). NF- $\kappa$ B and $\mathrm{I} \kappa \mathrm{B}$ are activated and shift into the nucleus from the cytoplasm (24). Studies have found that $\mathrm{NF}-\kappa \mathrm{B}$ is activated in rats with SCP at an early stage $(23,25)$. Activated $N F-\kappa B$ can be found in the cytoplasm and cell nuclei of neurons $24 \mathrm{~h}$ following injury (25). During the acute inflammatory reaction, $\mathrm{NF}-\kappa \mathrm{B}$ is involved in the activation of macrophages and hemameba, and controls the genetic expression of proinflammatory factors (25). The control of this process leads to the amplification of inflammatory responses and tissue injury (26). The present study observed that tanshinone IIA significantly suppressed the protein expression of NF- $\kappa \mathrm{B}$ and increased the protein expression of $\mathrm{p}-\mathrm{I} \kappa \mathrm{B}$ in the SCP rats. Bai et al also suggested that tanshinone IIA induces apoptosis through the suppression of $\mathrm{NF}-\kappa \mathrm{B}$ signaling in colon cancer cells (27).

SCP is one of the main factors resulting in neonatal death, which is closely associated with SCP (28). VEGF functions in promoting endothelial cell proliferation, and increasing vascular permeability and angiogenesis (28). It has been suggested that VEGF may have direct protective functions on endothelial cells and astrocytes of the central nervous system. It can promote angiogenesis, and stimulate axon growth and neuronal survival $(9,29)$. The present study demonstrated that tanshinone IIA significantly induced the protein expression of VEGF in SCP rats. Xu et al reported that tanshinone IIA protected free flaps against hypoxic injury through VEGF and CD34 in epithelial skin cells (30).

The activities of p38MAPK are significantly increased in microglial cells (31). Activated p38MAPK locates in the nucleus or endochylema, which may be associated with the functions and status of microglial cells (32). p38MAPK is associated with activities of NF- $\kappa \mathrm{B}$. The inhibition of p38MAPK can inhibit the transcription of $\mathrm{NF}-\kappa \mathrm{B}$ (33). Major biological functions following the activation of p38MAPK include generating and activating various inflammatory cytokines, including TNF- $\alpha$, IL-1 $\beta$, IL-6 and IL-8 (34). Macrophages at the ischemic core can be found with activated P38MAPK, indicating that p38MAPK may be involved in the inflammatory responses when cerebral ischemic injury occurs (32). In the present study, it was found that tanshinone IIA significantly suppressed the protein expression of p-p38 in SCP rats. Liu et al reported that the neuroprotective effects of tanshinone IIA significantly suppressed the protein expression of p-p38 and induced the protein expression of VEGF in SCP rats (35).

In conclusion, tanshinone IIA significantly inhibited the searching time and number of mistakes made by the rats, and increased the number of repetitions and holding times in the SCP rats. Tanshinone IIA also weakened the increased expression levels of TNF- $\alpha$, IL-1 $\beta$ and IL- 6 , suppressed the expression levels of MCP-1 and COX-2, reduced the protein expression of iNOS, and induced the protein expression of $n N O S$ in the SCP rats, which regulated the $N F-\kappa B$ and p38MAPK signaling pathway.

\section{References}

1. Kerkum YL, Buizer AI, van den Noort JC, Becher JG, Harlaar J and Brehm MA: The effects of varying ankle foot orthosis stiffness on gait in children with spastic cerebral palsy who walk with excessive knee flexion. PLoS One 10: e0142878, 2015.

2. Kuypers E, Ophelders D, Jellema RK, Kunzmann S, Gavilanes AW and Kramer BW: White matter injury following fetal inflammatory response syndrome induced by chorioamnionitis and fetal sepsis: Lessons from experimental ovine models. Early Hum Dev 88: 931-936, 2012.

3. Colson SB, Siparsky GL, Capocelli KE, Pan Z, Sokol RJ and Hoffenberg EJ: Inflammatory bowel disease in pediatric patients with cerebral palsy. J Pediatr Gastroenterol Nutr 56: e50, 2013.

4. Qi YC, Xiao XJ, Duan RS, Yue YH, Zhang XL, Li JT and Li YZ Effect of acupuncture on inflammatory cytokines expression of spastic cerebral palsy rats. Asian Pac J Trop Med 7: 492-495, 2014.

5. Beloosesky R, Ginsberg Y, Khatib N, Maravi N, Ross MG, Itskovitz-Eldor J and Weiner Z: Prophylactic maternal $\mathrm{N}$-acetylcysteine in rats prevents maternal inflammation-induced offspring cerebral injury shown on magnetic resonance imaging. Am J Obstet Gynecol 208: 213 e1-6, 2013.

6. Jacobsson B: Infectious and inflammatory mechanisms in preterm birth and cerebral palsy. Eur J Obstet Gynecol Reprod Biol 115: 159-160, 2004.

7. Destot-Wong KD, Liang K, Gupta SK, Favrais G, Schwendimann L, Pansiot J, Baud O, Spedding M, Lelièvre V, Mani S and Gressens P: The AMPA receptor positive allosteric modulator, S18986, is neuroprotective against neonatal excitotoxic and inflammatory brain damage through BDNF synthesis. Neuropharmacology 57: 277-286, 2009. 
8. Zheng XR, Zhang SS, Yin F, Tang JL, Yang YJ, Wang X and Zhong L: Neuroprotection of VEGF-expression neural stem cells in neonatal cerebral palsy rats. Behav Brain Res 230: 108-115, 2012.

9. El Ghazi F, Desfeux A, Brasse-Lagnel C, Roux C, Lesueur C Mazur D, Remy-Jouet I, Richard V, Jégou S, Laudenbach V, et al: NO-dependent protective effect of VEGF against excitotoxicity on layer VI of the developing cerebral cortex. Neurobiol Dis 45: 871-886, 2012

10. Lin JY, Ke YM, Lai JS and Ho TF: Tanshinone IIA enhances the effects of TRAIL by downregulating survivin in human ovarian carcinoma cells. Phytomedicine 22: 929-938, 2015.

11. Zhang K, Li J, Meng W, Xing H and Yang Y: Tanshinone IIA inhibits acute promyelocytic leukemia cell proliferation and induces their apoptosis in vivo. Blood Cells Mol Dis 56: 46-52, 2016.

12. Lu BL, Li J, Zhou J,Li WW and Wu HF: Tanshinone IIA decreases the levels of inflammation induced by Abeta1-42 in brain tissues of Alzheimer's disease model rats. Neuroreport 27: 883-893, 2016.

13. Qiu S, Granet R, Mbakidi JP, Brégier F, Pouget C, Micallef L, Sothea-Ouk T, Leger DY, Liagre B, Chaleix V and Sol V: Delivery of tanshinone IIA and $\alpha$-mangostin from gold/PEI/cyclodextrin nanoparticle platform designed for prostate cancer chemotherapy. Bioorg Med Chem Lett 26: 2503-2506, 2016.

14. Huetz N, Triau S, Leboucher B, Sentilhes L, Hanf M, Nguyen S, Flamant C, Roze JC and Gascoin G: Association of severe placental inflammation with death prior to discharge and cerebral palsy in preterm infants. BJOG 123: 1956-1963, 2016.

15. Srivastava IN, Shperdheja J, Baybis M, Ferguson T and Crino PB: mTOR pathway inhibition prevents neuroinflammation and neuronal death in a mouse model of cerebral palsy. Neurobiol Dis 85: 144-154, 2016.

16. Goldenberg RL and McClure EM: Placental inflammation, neonatal death and cerebral palsy in preterm infants: Is there a relationship? BJOG 123: 1964, 2016

17. Jiang C, Zhu W, Shao Q, Yan X, Jin B, Zhang M and Xu B: Tanshinone IIA protects against folic acid-induced acute kidney injury. Am J Chin Med 44: 737-753, 2016.

18. Lloyd E, Somera-Molina K, Van Eldik LJ, Watterson DM and Wainwright MS: Suppression of acute proinflammatory cytokine and chemokine upregulation by post-injury administration of a novel small molecule improves long-term neurologic outcome in a mouse model of traumatic brain injury. J Neuroinflammation 5 : 28,2008 .

19. Ragin AB, Wu Y, Storey P, Cohen BA, Edelman RR and Epstein LG: Monocyte chemoattractant protein-1 correlates with subcortical brain injury in HIV infection. Neurology 66 : 1255-1257, 2006

20. Helmy A, Guilfoyle MR, Carpenter KL, Pickard JD, Menon DK and Hutchinson PJ: Recombinant human interleukin-1 receptor antagonist promotes M1 microglia biased cytokines and chemokines following human traumatic brain injury. J Cereb Blood Flow Metab 36: 1434-1448, 2016.

21. Shein SL, Shellington DK, Exo JL, Jackson TC, Wisniewski SR, Jackson EK, Vagni VA, Bayır H, Clark RS, Dixon CE, et al Hemorrhagic shock shifts the serum cytokine profile from proto anti-inflammatory after experimental traumatic brain injury in mice. J Neurotrauma 31: 1386-1395, 2014.

22. Tang J, Zhu C, Li ZH, Liu XY, Sun SK, Zhang T, Luo ZJ, Zhang H and Li WY: Inhibition of the spinal astrocytic JNK/MCP-1 pathway activation correlates with the analgesic effects of tanshinone IIA sulfonate in neuropathic pain. J Neuroinflammation 12: 57, 2015.
23. Wang ZR, Li YX, Lei HY, Yang DQ, Wang LQ and Luo MY: Regulating effect of activated NF- $\mathrm{KB}$ on edema induced by traumatic brain injury of rats. Asian Pac J Trop Med 9: 274-277, 2016.

24. Su X, Wang H, Zhao J, Pan H and Mao L: Beneficial effects of ethyl pyruvate through inhibiting high-mobility group box 1 expression and TLR4/NF- $\kappa \mathrm{B}$ pathway after traumatic brain injury in the rat. Mediators Inflamm 2011: 807142, 2011.

25. Wang CX, Xie GB, Zhou CH, Zhang XS, Li T, Xu JG, Li N, Ding K, Hang CH, Shi JX and Zhou ML: Baincalein alleviates early brain injury after experimental subarachnoid hemorrhage in rats: Possible involvement of TLR4/NF- $\mathrm{BB}$-mediated inflammatory pathway. Brain Res 1594: 245-255, 2015.

26. Gao W, Zhao Z, Yu G, Zhou Z, Zhou Y, Hu T, Jiang R and Zhang J: VEGI attenuates the inflammatory injury and disruption of blood-brain barrier partly by suppressing the TLR4/NF- $\kappa \mathrm{B}$ signaling pathway in experimental traumatic brain injury. Brain Res 1622: 230-239, 2015.

27. Bai Y, Zhang L, Fang X and Yang Y: Tanshinone IIA enhances chemosensitivity of colon cancer cells by suppressing nuclear factor- $\kappa$ B. Exp Ther Med 11: 1085-1089, 2016.

28. Dashti SR, Spalding A, Kadner RJ, Yao T, Kumar A, Sun DA and LaRocca R: Targeted intraarterial anti-VEGF therapy for medically refractory radiation necrosis in the brain. J Neurosurg Pediatr 15: 20-25, 2015.

29. Jin X, Liang B, Chen Z, Liu X and Zhang Z: The dynamic changes of capillary permeability and upregulation of VEGF in rats following radiation-induced brain injury. Microcirculation 21: 171-177, 2014

30. Xu Z, Wu L, Sun Y, Guo Y, Qin G, Mu S, Fan R, Wang B, Gao W and Zhang Z: Tanshinone IIA pretreatment protects free flaps against hypoxic injury by upregulating stem cell-related biomarkers in epithelial skin cells. BMC Complement Altern Med 14: 331, 2014

31. Choi DC, Lee JY, Lim EJ, Baik HH, Oh TH and Yune TY: Inhibition of ROS-induced p38MAPK and ERK activation in microglia by acupuncture relieves neuropathic pain after spinal cord injury in rats. Exp Neurol 236: 268-282, 2012.

32. Yang H, Feng GD, Liang Z, Vitale A, Jiao XY, Ju G and You SW: In vitro beneficial activation of microglial cells by mechanically-injured astrocytes enhances the synthesis and secretion of BDNF through p38MAPK. Neurochem Int 61: 175-186, 2012

33. Lee KF, Chen JH, Teng CC, Shen $\mathrm{CH}$, Hsieh MC, Lu CC, Lee KC, Lee LY, Chen WP, Chen CC, et al: Protective effects of Hericium erinaceus mycelium and its isolated erinacine A against ischemia-injury-induced neuronal cell death via the inhibition of iNOS/p38 MAPK and nitrotyrosine. Int J Mol Sci 15: 15073-15089, 2014.

34. Su J, Zhou H, Tao Y, Guo J, Guo Z, Zhang S, Zhang Y, Huang Y, Tang Y, Dong Q and Hu R: G-CSF protects human brain vascular endothelial cells injury induced by high glucose, free fatty acids and hypoxia through MAPK and Akt signaling. PLoS One 10: e0120707, 2015.

35. Liu X, Ye M, An C, Pan L and Ji L: The effect of cationic albumin-conjugated PEGylated tanshinone IIA nanoparticles on neuronal signal pathways and neuroprotection in cerebral ischemia. Biomaterials 34: 6893-6905, 2013. 